\title{
Drug-Drug Interactions between Antiarrhythmic Drugs in Chick Embryos
}

\author{
Yuji Yoshiyama, ${ }^{*, a}$ Takashi SugiYama, ${ }^{b}$ Motoko Kanke, ${ }^{a}$ and Kanji Tsuchimoto ${ }^{b}$ \\ ${ }^{a}$ Division of Clinical Pharmacy, Kyoritsu College of Pharmacy; 1-5-30 Shibakoen, Minato-ku, Tokyo 105-8512, Japan: \\ and ${ }^{b}$ Division of Pathophysiology, Center for Clinical Pharmacy and Clinical Sciences, School of Pharmaceutical \\ Sciences, Kitasato University; Minato-ku, Tokyo 108-8641, Japan. \\ Received July 31, 2003; accepted September 12, 2003
}

The drug-drug interactions between antiarrhythmic drugs were studied in chick embryos. Fertilized eggs of White Leghorns were incubated and investigated. Procainamide or flecainide with and without propranolol was injected into the air sac of a fertilized egg. Electrocardiograms (ECGs) were recorded 0 to $60 \mathrm{~min}$ after the injection. After each drug injection alone, the heart rate was not different compared with the control. However, the heart rate was significantly decreased by combinations of procainamide and propranolol or flecainide and propranolol. In addition, arrhythmia was produced in combination with propranolol. These findings indicate that the drug-drug interactions between antiarrhythmic drugs have a marked influence on the heart rate in chick embryos.

Key words drug-drug interaction; procainamide; flecainide; propranolol; chick embryo; electrocardiogram

Many antiarrhythmic drugs have been put on the market in Japan and classified into subcategories. Such classification of antiarrhythmic mechanisms should prove of value in predicting the therapeutic as well as toxic effects of different agents, and may be particularly important in planning combination therapy for resistant arrhythmias. Recently, since reports of the Cardiac Arrhythmia Suppression Trial (CAST), proarrhythmic actions induced by antiarrhythmic drugs have been widely studied. ${ }^{1,2)}$

We have evaluated the proarrhythmic actions of antiarrhythmic drugs in chick embryos. ${ }^{3)}$

With the recent concern for animal rights, experimental studies using mammals have been limited in number and methods. Thus, based on social acceptance, experimental studies using chick embryos have drawn attention. Chick embryos have been widely used in pharmacological and toxicological experiments for evaluating drug action on the fetus. ${ }^{4-6)}$ Chick embryonic heart develops through a similar process to that of mice, rats and humans, and also has a similar atrioventricular system (Bulter and Juurlink, 1987).

In order to develop alternative research methods, we have studied the biological effects of drugs on the cardiovascular system of chick embryos using physiological techniques. ${ }^{7-11)}$

Drug-drug interactions have been demonstrated for a variety of drugs in the treatment of heart failure patients. ${ }^{12,13)} \mathrm{We}$ have previously evaluated the toxic interactions between propranolol and disopyramide in chick embryos. ${ }^{14,15)}$

The present study evaluated the drug-drug interactions between antiarrhythmic drugs in chick embryos.

\section{MATERIALS AND METHODS}

Eggs and Incubation Fertilized eggs of White Leghorns (Omiya Poultry Laboratory, Saitama, Japan) were incubated at $37.5 \pm 0.2^{\circ} \mathrm{C}$ at a relative humidity of about $65 \%$, turned automatically every hour, and candled daily to check their viability.

Antiarrhythmic Drugs Used Procainamide hydrochloride (Daiichi Pharmaceutical Co., Ltd., Tokyo), flecainide acetate (Eisai Co., Ltd., Tokyo) and propranolol (Sumitomo
Pharmaceutical, Tokyo, Japan) were purchased from commercial sources and were diluted with sterilized physiological saline to the desired concentrations in $0.2 \mathrm{ml}$ of solution.

Injection of Drugs and Electrocardiogram (ECG) Recording in Chick Embryos Anesthetic injection of urethane $([45 \mathrm{mg} / \mathrm{egg}]+\alpha$-chloralose $[4.5 \mathrm{mg} / \mathrm{egg}])$ was performed into the air sac of eggs to record the stable ECG waves in 16-d-old embryos in which $\mathrm{R} R$ intervals could be clearly analyzed. Drugs were injected into the air sac of eggs in a volume of $0.2 \mathrm{ml} 20 \mathrm{~min}$ after injection of the anesthetic. Procainamide at $3 \mathrm{mg} / \mathrm{egg}$, flecainide at $0.3 \mathrm{mg} / \mathrm{egg}$ and propranolol at $0.01 \mathrm{mg} / \mathrm{egg}$ were injected into the air sac of each fertilized egg on the 16th day of incubation. After each drug injection alone or in combination, procainamide and propranolol or flecainide and propranolol, the values of heart rate were measured.

ECGs were recorded 0 to $60 \mathrm{~min}$ after the drug injection, and heart rate was determined from R R intervals. Data represented the percentage of changes in the heart rate from each 0 time.

Four small holes every 90 degrees on "the equator", one small hole on "the south pole" and one small hole on "the north pole" were made on each fertilized egg by an electric drill and sealed with Paraffin (mp $60^{\circ} \mathrm{C}$ ). Specially designed needle electrodes were inserted into the appropriate holes of "the equator" and "the south pole". The two needles on "the equator" were used as a bipolar lead of the embryonic heart, and the needle on "the south pole" was used as a ground lead. These needles were connected to the memory oscilloscope (VC-11, Nihon Koden Co., Tokyo, Japan). ECGs were recorded as bipolar waves between two needles on a recorder (PowerLab System, ADInstruments Japan Co., Tokyo, Japan) (Fig. 1).

The data were analyzed by one way analysis of variance. If there was a significant difference among the groups, a multiple comparison test was conducted (Tukey's test). The fiducial limit of 0.05 , two-tails, was used as the criterion to determine significance. 


\section{RESULTS}

The body weight of chick embryos gradually increased with the day of incubation. After each drug injection alone, the values of heart rate were not different compared with the

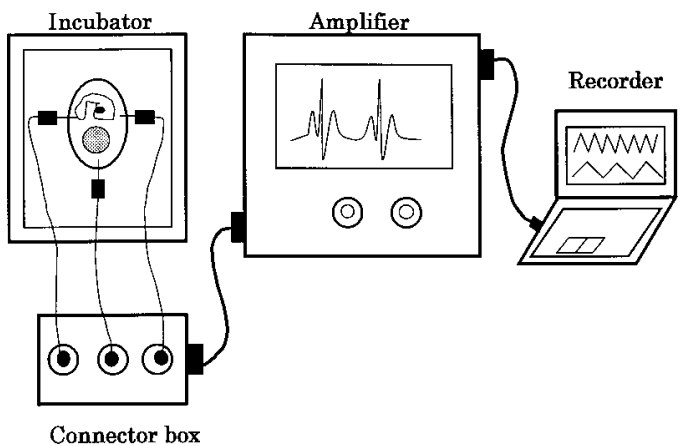

Fig. 1. Schema of ECG-Recording System for Chick Embryo in Egg Shell control. However, the heart rate was significantly decreased by combination with procainamide and propranolol or flecainide and propranolol (Figs. 2, 3). In addition, arrhythmia was produced in combination with propranolol (Fig. 4).

\section{DISCUSSION}

We have reported that chick embryos treated with antiarrhythmic drugs exhibit proarrythmic effects. ${ }^{3)}$ When the ECG waves in the treated chick embryos were anlyzed, various ECG wave cycles, including arrhythmias, PQ, QRS and QT prolongations were observed, depending on the drug used. ${ }^{3)}$ The P, QRS and T waves were noted in the ECG tracing from the chick embryos. Among the wave intervals, the QT interval, rather than the QRS and PQ intervals, was positively correlated to the $\mathrm{R} R$ interval, as in humans and canines, suggesting the usefulness of the in ovo anesthetized chick embryos for physiological and pharmacological studies. ${ }^{12)}$ This study was designed to identify changes in heart

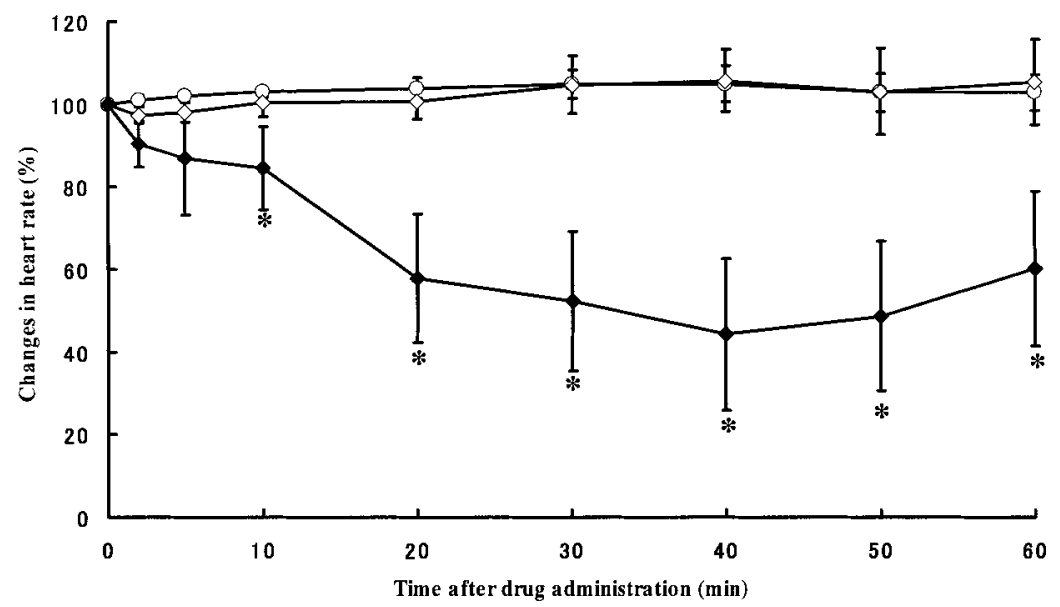

Fig. 2. Changes in Heart Rate of Chick Embryo after Administration of Procainamide in Combination with Propranolol

Procainamide $3 \mathrm{mg} / \mathrm{egg}$ alone $(\diamond)$, propranolol $0.01 \mathrm{mg} / \mathrm{egg}$ alone $(\bigcirc)$ or procainamide $3 \mathrm{mg} / \mathrm{egg}$ plus propranolol $0.01 \mathrm{mg} / \mathrm{egg}(\diamond)$ was injected into the air sac of fertile eggs on the 16th day of incubation. Each point represents the mean and standard deviation for six eggs. $*$ Significantly different from the procainamide $3 \mathrm{mg} / \mathrm{egg}$ alone group, $p<0.05$. The heart rates of chick embryos before each drug injection; procainamide $3 \mathrm{mg} / \mathrm{egg}$ alone: $218 \pm 9$ beats/min, propranolol $0.01 \mathrm{mg} / \mathrm{egg}$ alone: $228 \pm 13$ beats/min, procainamide $3 \mathrm{mg} / \mathrm{egg}$ plus propranolol $0.01 \mathrm{mg} / \mathrm{egg}: 230 \pm 9$ beats $/ \mathrm{min}$

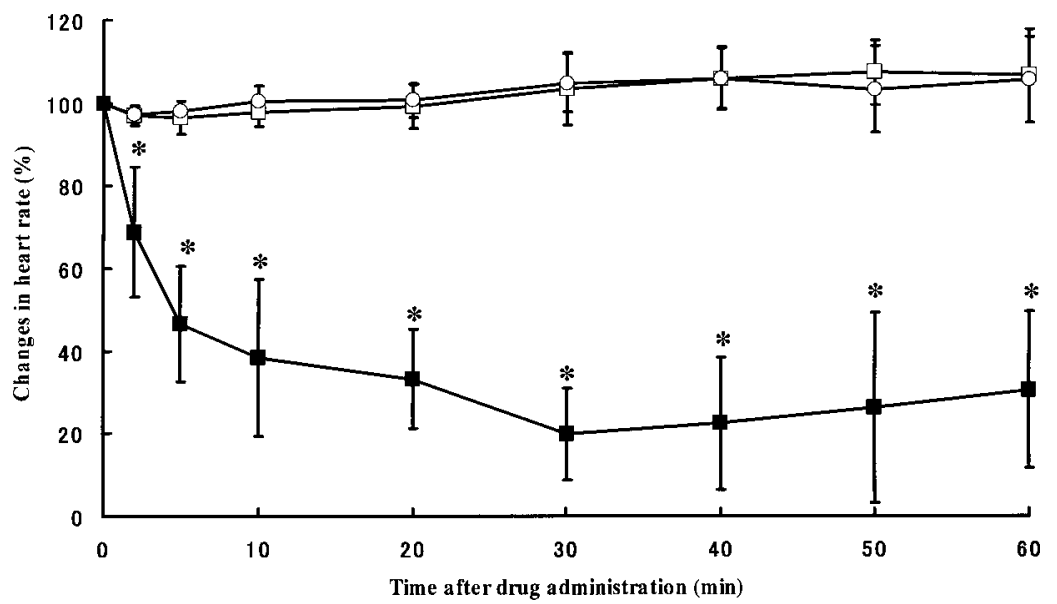

Fig. 3. Changes in Heart Rate of Chick Embryo after Administration of Flecainide in Combination with Propranolol

Flecainide $0.3 \mathrm{mg} / \mathrm{egg}$ alone $(\square)$, propranolol $0.01 \mathrm{mg} / \mathrm{egg}$ alone $(\bigcirc)$ or flecainide $0.3 \mathrm{mg} / \mathrm{egg}$ plus propranolol $0.01 \mathrm{mg} / \mathrm{egg}(\boldsymbol{\square})$ was injected into the air sac of fertile eggs on the 16 th day of incubation. Each point represents the mean and standard deviation for six eggs. * Significantly different from the flecainide $0.3 \mathrm{mg} / \mathrm{egg}$ alone group, $p<0.05$. The heart rates of chick embryos before each drug injection; flecainide $0.3 \mathrm{mg} / \mathrm{egg}$ alone: $212 \pm 13$ beats $/ \mathrm{min}$, propranolol $0.01 \mathrm{mg} / \mathrm{egg}$ alone: $228 \pm 13 \mathrm{beats} / \mathrm{min}$, flecainide $0.3 \mathrm{mg} / \mathrm{egg}$ plus propranolol $0.01 \mathrm{mg} / \mathrm{egg}$ : $222 \pm 9$ beats $/ \mathrm{min}$ 


\section{$0 \mathrm{~min}$}

\section{$60 \mathrm{~min}$}

A:

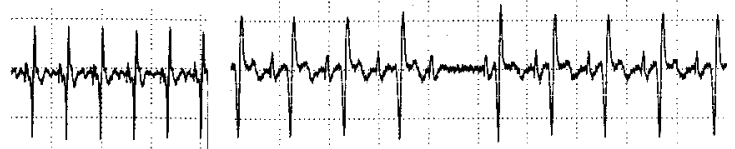

B:

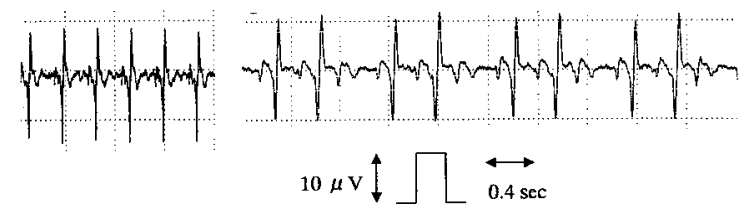

Fig. 4. Arrhythmias in Chick Embryos Treated with Procainamide or Flecainide with Propranolol

A: Procainamide $3 \mathrm{mg} / \mathrm{egg}$ plus propranolol $0.01 \mathrm{mg} / \mathrm{egg}$. B: Flecainide $0.3 \mathrm{mg} / \mathrm{egg}$ plus propranolol $0.01 \mathrm{mg} / \mathrm{egg}$.

rate which decrease in a dose-response manner after treatment.

After each drug injection alone, the heart rate of the chick embryos was not different compared with the control. Toxic interactions between antiarrhythmic agents were demonstrated in chick embryos. The combination with propranolol modified the pharmacological effects of the antiarrhythmic agents in chick embryos and led to a Q Tc interval prolongation of the ECGs. It is surprising that propranolol, which has long been noted for its negative inotropic myocardial action, has been reported to cause little heart failure in patients. ${ }^{13)}$

After the drug was injected into the air sac of each fertilized egg, it accumulated in the egg shell. Therefore, the heart rate may be decreased time-dependently. This time-dependent effect of the drug on the heart rate should be investigated further.

We have demonstrated in this report that our recording system for an electron cardiogram system using chick embryo may be applied as an animal test alternative.

Accordingly, this toxic interaction could be clearly analyzed in chick embryos, as in mammals, especially the decrease in heart rate, namely, prolongation of the $\mathrm{R} R$ interval, accompanied by prolongation of the $\mathrm{Q}$ Tc interval in the chick embryos, as was observed in humans. The clinical use of antiarrhythmic agents in conjunction with other antiarrhythmic agents, such as propranolol, led to Q Tc interval prolongation of the ECGs. ${ }^{13)}$ This indicates that developing chick embryos are appropriate as an alternative experimental animal rather than traditional mammals.

Nayler has shown that propranolol inhibits the lipid-facilitated transport of calcium from an aqueous to a lipid-solvent phase. Such an interaction may inhibit or impede the transport of calcium from the sarcoplasmic reticulum through lipid membranes and cause a reduced concentration of myoplasmic calcium that is inadequate for the proper initiation of contraction, thereby resulting in myocardial depression. ${ }^{17)}$

Although the exact mechanism underlying the influence of the interaction on the pharmacological effects of the drug remains to be clarified, the interaction seems to enhance the toxicity of the drug in chick embryos.

In conclusion, our in ovo recording system for ECG of chick embryos may be useful for investigating toxic interactions among antiarrhythmic drugs.

\section{REFERENCES}

1) Cardiac Arrhythmia Suppression Trial (CAST) Investigators, N. Engl. J. Med., 321, 406-412 (1989).

2) CAST II Investigators, N. Engl. J. Med., 327, 227-233 (1992).

3) Sugiyama T., Miyazaki H., Shimada H., AATEX, 6, $72-78$ (1999).

4) Tazawa H., Yamaguchi S., Yamada M., Doi K., Comp. Biochem. Physiol., 101A, 103-108 (1992).

5) Rajala G. M., Kuhlmann R. S., Kolesari G. L., Teratology, 30, 385392 (1984).

6) Paff G. H., Boucek R. J., Anat. Rec., 182, 169-173 (1975).

7) Bulter H., Juurlink B. H. J., "An Atlas for Staging Mammalian and Chick Embryos,” CRC Press Inc., Boca Raton, 1987, pp. 19-138.

8) Yoshiyama Y., Sugiyama T., Kanke M., Biol. Pharm. Bull., 26, 893895 (2003).

9) Yoshiyama Y., Sugiyama T., Kanke M., AATEX, 9, 40-44 (2003).

10) Miyazaki H., Sugiyama T., Saito K., Kubota N., Yoshiyama Y., Shimada H., In Vitro Toxicol., 7, 243-246 (1994).

11) Saito K., Sugiyama T., Shimada H., Eur. J. Pharmacol., 183, 22362237 (1990)

12) Sugiyama T., Miyazaki H., Saito K., Shimada H., Miyamoto K., Toxicol. Appl. Pharmacol., 138, 262-267 (1996).

13) Ellrodt G., Singh B. N., Heart Lung, 9, 469-474 (1980).

14) Podrid P. J., Schoenberger A., Lown B., N. Engl. J. Med., 302, 614617 (1980).

15) Yoshiyama Y., Sugiyama T., Miyazaki H., Kanke M., Shimada H., Biol. Pharm. Bull., 20, 1310-1312 (1997).

16) Yoshiyama Y., Sugiyama T., Kanke M., Tsuchimoto K., Biol. Pharm. Bull., 24, 429-431 (2001).

17) Nayler W. G., J. Pharmacol. Exp. Ther., 153, 479-484 (1966). 